\title{
COVID-19 vaccine uptake among healthcare workers in the fourth country to authorize BNT162b2 during the first month of rollout
}

\author{
Mazin Barry $^{\mathrm{a}, *, 1}$, Mohamad-Hani Temsah ${ }^{\mathrm{b}, 1}$, Fadi Aljamaan ${ }^{\mathrm{c}, \mathrm{f,g}}$, Basema Saddik ${ }^{\mathrm{h}, \mathrm{i}}$, Ayman Al-Eyadhy ${ }^{\mathrm{b}}$, \\ Shuliweeh Alenezi ${ }^{\circ}$, Nurah Alamro ${ }^{c}$, Abdullah N Alhuzaimi ${ }^{\mathrm{c}, \mathrm{d}, \mathrm{e}}$, Ali Alhaboob ${ }^{\mathrm{b}}$, Khalid Alhasan ${ }^{\mathrm{b}}$, \\ Fahad Alsohime $^{\mathrm{b}}$, Ali Alaraj ${ }^{\mathrm{g}, \mathrm{j}}$, Rabih Halwani ${ }^{\mathrm{h}, \mathrm{k}}$, Amr Jamal ${ }^{\mathrm{c}, \mathrm{l}, \mathrm{m}}$, Omar Temsah ${ }^{\mathrm{n}}$, Fahad Alzamil ${ }^{\mathrm{b}}$, \\ Ali Somily ${ }^{\mathrm{c}}$, Jaffar A. Al-Tawfiq ${ }^{\mathrm{p}, \mathrm{q}, \mathrm{I}}$
}

${ }^{a}$ Division of Infectious Diseases, Department of Internal Medicine, College of Medicine, King Saud University and King Saud University Medical City, Riyadh, Saudi Arabia

${ }^{\mathrm{b}}$ Pediatric Department, College of Medicine, King Saud University, Riyadh, Saudi Arabia

${ }^{\mathrm{c}}$ College of Medicine, King Saud University, Riyadh, Saudi Arabia

${ }^{\mathrm{d}}$ Division of Pediatric Cardiology, Department of Cardiac Sciences, King Saud University Medical City, College of Medicine, King Saud University, Riyadh, Saudi Arabia

${ }^{\mathrm{e}}$ Heart Center, King Faisal Specialist Hospital \& Research Center, Riyadh, Saudi Arabia

${ }^{\mathrm{f}}$ Critical Care Department, College of Medicine, King Saud University, Riyadh, Saudi Arabia

${ }^{g}$ Dr. Sulaiman Al Habib Medical Group, Riyadh, Saudi Arabia

${ }^{\mathrm{h}}$ Sharjah Institute of Medical Research, University of Sharjah, Sharjah, United Arab Emirates

${ }^{\mathrm{i}}$ Department of Family and Community Medicine, College of Medicine, University of Sharjah, Sharjah, United Arab Emirates

${ }^{\mathrm{j}}$ Department of Medicine, College of Medicine, Qassim University, Qassim, Saudi Arabia

${ }^{\mathrm{k}}$ Department of Clinical Sciences, College of Medicine, University of Sharjah, Sharjah, United Arab Emirates

${ }^{1}$ Department of Family and Community Medicine, King Saud University Medical City, Riyadh, Saudi Arabia

${ }^{\mathrm{m}}$ Evidence-Based Health Care \& Knowledge Translation Research Chair, King Saud University, Riyadh, Saudi Arabia

${ }^{n}$ College of Medicine, Alfaisal University, Riyadh, Saudi Arabia

${ }^{\circ}$ Department of Psychiatry, College of Medicine, King Saud University, Riyadh, Saudi Arabia

${ }^{\mathrm{P}}$ Specialty Internal Medicine and Quality Department, Johns Hopkins Aramco Healthcare, Dhahran, Saudi Arabia

${ }^{\mathrm{q}}$ Infectious Disease Division, Department of Medicine, Indiana University School of Medicine, Indianapolis, IN, USA

${ }^{\mathrm{r}}$ Infectious Disease Division, Department of Medicine, Johns Hopkins University School of Medicine, Baltimore, MD, USA

\section{A R T I C L E I N F O}

\section{Article history:}

Received 30 January 2021

Received in revised form 15 August 2021

Accepted 24 August 2021

Available online $\mathrm{xxxx}$

\section{Keywords:}

COVID-19

BNT162b2

Vaccine uptake

Healthcare workers

Kingdom of Saudi Arabia

\begin{abstract}
A B S T R A C T
Background: The Kingdom of Saudi Arabia (KSA) was the fourth country in the world to authorize the BNT162b2 coronavirus disease 2019 (COVID-19) vaccine, which it rolled out on December 17, 2020 and first targeted at healthcare workers (HCWs). This study assesses vaccine uptake among this group during the first month of its availability.

Methods: A national cross-sectional, pilot-validated, self-administered survey was conducted among HCWs in the KSA between December 27, 2020 and January 3, 2021. The survey included sociodemographic details, previous contact with COVID-19 patients, previous infection with COVID-19, receiving (or registering with the Ministry of Health website to receive) the COVID-19 vaccine, sources of HCWs' information on vaccines, awareness of emerging variants of concern, and anxiety level using the 7item Generalized Anxiety Disorder assessment. A descriptive bivariate analysis and multivariate logistic binary regression analysis were performed. The primary evaluated outcome was vaccine uptake.

Results: Of the 1058 participants who completed the survey, 704 (66.5\%) were female, and 626 (59.2\%) were nurses. Of all the respondents, 352 (33.27\%) were enrolled to receive or had already received the vaccine, while 706 (66.73\%) had not enrolled. In a bivariate analysis, not enrolling for vaccination was more likely in females than males (78.5\% vs. 21.5\%, P < 0.001), HCWs between the ages of 20 and 40 years than those $>40$ years $(70.4 \%$ vs. $29.6 \%, \mathrm{P}=0.005)$, Saudi HCWs than expatriates ( $78 \%$ vs $22 \%, \mathrm{P}<0.001)$, and among HCWs who used social media as a source of information than those who did not (69.8\% vs. $38.6 \%, \mathrm{P}<0.001)$. In a multivariate analysis, independent factors associated with uptake were being a Saudi national $(\mathrm{aOR}=1.918,95 \% \mathrm{CI}=1.363-2.698, \mathrm{P}<0.001)$, working in an intensive care unit $(\mathrm{aOR}=1.495,95 \% \mathrm{CI}=1.083-2.063, \mathrm{P}=0.014)$, and working at a university hospital $(\mathrm{aOR}=1.867,95 \%$ $\mathrm{CI}=1.380-2.525, \mathrm{P}<0.001)$.
\end{abstract}

\footnotetext{
* Corresponding author.

E-mail address: mbarry@ksu.edu.sa (M. Barry).
}

1 These authors contributed equally to this research. 
Conclusions: A low level of vaccine uptake was observed especially in female HCWs, those younger than 40 years old, and those who used social media as their source of vaccine information. This survey provides important information for public health authorities in order to scale up vaccination campaigns targeting these HCWs to increase vaccine enrollment and uptake.

(c) 2021 The Authors. Published by Elsevier Ltd. This is an open access article under the CC BY license (http:// creativecommons.org/licenses/by/4.0/).

\section{Introduction}

After the coronavirus disease 2019 (COVID-19) reached pandemic levels, vaccine development was fast tracked through government funding, corporate spending, and private donations [1]. Once vaccines were made available in December 2020, a phased approach for vaccine allocation was recommended, with Phase 1a targeting first respondents and healthcare workers (HCWs) [2]. Several vaccine manufacturers have published their Phase 3 trials confirming the safety and efficacy of the vaccine [3-5]. However, such unprecedented scientific achievement is challenged by the hesitancy of HCWs to accept vaccination [6]. In an earlier study from the Kingdom of Saudi Arabia (KSA), 70\% of the 1521 HCWs surveyed were willing to receive the COVID-19 vaccine [6]. Another study showed that $63 \%$ of the nurses surveyed were willing to receive the COVID-19 vaccine [6,7]. In two studies, the acceptance of the COVID-19 vaccine among adults was found to be between $58 \%$ and $69 \%$ [8,9]. The first case of COVID-19 in KSA was detected on March 2, 2020, and as of December 28, 2020, a total of 362,972 laboratory-confirmed cases were reported with 6239 deaths [10]. The KSA granted Pfizer/BioNTech emergency use authorization for the BNT162b2 vaccine on December 10, 2020, becoming the fourth country to do so after the United Kingdom (UK) that approved it December 2, Bahrain December 4, and Canada December 9, 2020 [11,12], As of January 3, 2021, at least one dose of vaccine was given to 1,380,430 individuals in UK, 60,097 in Bahrain, and 114,034 in Canada, no data was reported specifically on HCWs [13]. On that same day, the Ministry of Health $(\mathrm{MoH})$ sent out mass short message service texts and emails to all HCWs in the country encouraging them to voluntarily enroll for vaccine uptake through a dedicated smartphone application or the $\mathrm{MoH}$ website. COVID-19 vaccine rollout began on December 17,2020 . The number of healthcare professionals in KSA was estimated to be 350,000 in 2014 [14]. This study was conducted to evaluate vaccine enrollment and uptake within the first month of its rollout among HCWs in the KSA.

\section{Method}

\subsection{Data collection}

This national cross-sectional survey was conducted among HCWs in Saudi Arabia during the COVID-19 pandemic. Data were collected between December 27, 2020 and January 3, 2021. At the time of data collection, the national coronavirus vaccination campaign had already begun in the KSA, with HCWs as one of the prioritized groups. HCWs were surveyed regarding their intention to receive the COVID-19 vaccine. Participants were invited using a convenience sampling technique. We used several social media platforms and email lists to recruit participants. The survey was a pilot-validated, self-administered questionnaire (Supplementary appendix) that was sent to HCWs through SurveyMonkey $\odot$, a platform that allows researchers to deploy and analyze surveys via the web. The study utilized various methods to disseminate the survey questionnaires with dependence on most frequently used social platforms in Saudi Arabia, such as WhatsApp and Twitter and through the inclusion of various coauthors situ- ated in the different parts of the country. The questionnaire was adapted from our previously published studies with modification and additions related to the new severe acute respiratory syndrome coronavirus 2 (SARS-CoV-2) variant of concern (VoC) B.1.1.7 or alpha $[6,15-17]$.

The questions asked about respondents' demographic characteristics (job category, age, gender, years of clinical experience, and work area), previous exposure to COVID-19 patients, previous COVID-19 infection, and travel history in the prior 3 months. We assessed the level of intention to and actual receipt (i.e., uptake) of the COVID-19 vaccine among HCWs. In addition, we assessed factors affecting respondents' intention to receive the COVID-19 vaccine, including their level of awareness of the new SARS-CoV2 VoC B.1.1.7 and sources of information. HCWs' anxiety was measured by the validated 7-item General Anxiety Disorder (GAD-7) questionnaire, which has been used in several studies assessing HCWs' anxiety levels during the pandemic [16,18].

HCWs were informed of the purpose of the study in English at the beginning of the online survey. The respondents were given the opportunity to ask questions via a dedicated email address for the study. The Institutional Review Board at the College of Medicine and King Saud University Medical City approved the study (approval \#20/0065/IRB). A waiver for signed consent was obtained since the survey presented no more than a minimal risk to subjects and involved no procedures for which written consent is usually required. To maximize confidentiality, personal identifiers were not required. No financial incentives were provided.

\subsection{Statistical analyses}

Descriptive analyses with means and standard deviations were applied to continuous variables, and categorically measured variables were described with frequencies and percentages. Histograms and statistical Kolmogorov-Smirnov tests of normality were used to assess the statistical normality of continuous variables. HCWs' awareness of the new mutagenic COVID-19 virus strain B.1.1.7 was measured with eight questions, which received a score of 1 for each correctly answered knowledge/awareness question and 0 for each incorrectly answered question. Total awareness of the mutagenic viral outbreak was measured by adding up the total scores on the knowledge indicators, yielding a mutagenic disease awareness ranging from 0 to 8 points.

Independent samples t-tests were used to assess the statistical significance of mean scores between the levels of dichotomous categorical variables. Chi-squared tests of independence were used to assess the associations between categorically measured variables with the HCWs' uptake of the COVID-19 vaccine. The logistic binary regression analysis was used to understand HCWs' immunization uptake by regressing their sociodemographic, clinical, and professional characteristics and mutant viral strain perceptions against their odds of having actively received the COVID-19 immunization shot or registering for it. The associations between HCWs' measured independent variables and COVID-19 vaccine uptake were expressed as adjusted odds ratios (aOR) with 95\% confidence intervals $(95 \% \mathrm{CI})$. IBM $^{\circledR}$ SPSS $^{\circledR}$ was used for the statistical data analysis, and significance was considered at the 0.05 alpha level. 


\section{Results}

Out of 1212 HCWs who accessed the survey and consented to participate, 1058 (87.2\%) completed all survey questions and were included in the analysis, the majority were female $(n=704,66.5 \%)$, most of them were expatriates ( $n=600,85.2 \%$ ). The sociodemographic characteristics of all study participants are shown in Table 1. The eight questions regarding the VoC B.1.1.7 are listed in Table 2.

Of all the respondents, 352 (33.27\%) were enrolled to receive or had already received the vaccine, while 706 (66.73\%) did not wish to register for vaccination. The bivariate analysis of association between the respondents' characteristics and their tendency to receive the vaccine is shown in Table 3 . A significantly higher percentage of females compared to males reported not receiving or registering to receive the vaccine $(78.5 \%$ vs. $21.5 \%, \mathrm{P}<0.001)$, and younger age (between 20 and 40 years old) was associated with a significant tendency to decline to receive the vaccine compared to older age groups $(P=0.005)$. A lower percentage of expatriates reported receiving or registering to receive the vaccine compared to Saudi nationals $(\mathrm{P}<0.001)$. As for source of information on vaccines, the most frequent sources were social media (67\%), WHO website (39\%), MOH website (34\%), scientific journals (23.3\%) and CDC website (18.5\%).

A significantly higher percentage of nurses (69.5\%) and HCWs working in public/governmental hospitals (49.8\%) had not received or registered to receive the vaccine in comparison to other clinical roles $(\mathrm{P}<0.001)$ and HCWs in other hospital sectors $(\mathrm{P}=0.002)$. HCWs working in university hospitals $(44.9 \%, \mathrm{P}=0.002)$ and tertiary care hospitals $(74.1 \%, \mathrm{P}=0.048)$ were more inclined to receive the vaccine.

HCWs' previous infection with laboratory-confirmed COVID-19, previous contact with COVID-19 patients, and their travel history over the last 3 months were not correlated with their vaccine uptake. The respondents who were inclined to receive the vaccine were significantly less dependent on using social media as a source of information and had a significantly lower GAD-7 score, higher awareness about the new $\mathrm{VoC}$, and lower level of worry about travelling abroad.

A binomial logistic regression was performed to analyze the independent association between HCWs' characteristics and their vaccine uptake behavior as shown in Table 4. Females were significantly less likely to receive or register to receive the vaccine $(\mathrm{aOR}=0.287, \mathrm{P}<0.001)$, while older age $(\mathrm{aOR}=1.021, \mathrm{P}=0.032)$ and Saudi nationality $(\mathrm{aOR}=1.918, \mathrm{P}=0.001)$ were associated with an increased likelihood of vaccine uptake. Intensive care unit (ICU) staff $(\mathrm{aOR}=1.495, \mathrm{P}=0.014)$ and staff working in university hospitals $(\mathrm{aOR}=1.867, \mathrm{P}<0.001)$ were also significantly and independently more likely to receive or register to receive the vaccine. A higher level of awareness of the $\mathrm{VoC}$ also significantly predicted vaccine uptake among HCWs ( $\mathrm{aOR}=1.131, \mathrm{P}=0.047$ ). HCWs' level of anxiety as measured by their GAD-7 score, history of travelling abroad over the previous 3 months, and personal history of previous polymerase chain reaction (PCR)-positive COVID-19 did not independently predict their vaccine uptake behavior. Fig. 1 illustrates the linear incremental relation between HCWs' age and the probability of vaccine uptake, as the probability rose from about $30 \%$ for the $20-31$ age group to almost $45 \%$ for those over 50 years of age.

\section{Discussion}

In this reported national survey on COVID-19 vaccine uptake in one of the first countries to roll out the BNT162b2 vaccine, only 352 (33.3\%) of 1058 HCWs had either registered to/received the
Table 1

Descriptive analysis of HCWs' sociodemographic and professional characteristics $(\mathrm{N}=1058)$.

\begin{tabular}{|c|c|c|}
\hline Characteristic & $\mathrm{n}$ & $\%$ \\
\hline \multicolumn{3}{|l|}{ Gender } \\
\hline Male & 354 & 33.5 \\
\hline Female & 704 & 66.5 \\
\hline \multicolumn{3}{|l|}{ Age } \\
\hline 20-30 years & 238 & 22.5 \\
\hline $31-40$ years & 471 & 44.5 \\
\hline $41-50$ years & 263 & 24.9 \\
\hline$\geq 51$ years & 86 & 8.1 \\
\hline \multicolumn{3}{|l|}{ Marital state } \\
\hline Single & 257 & 24.3 \\
\hline Married & 769 & 72.7 \\
\hline Divorced & 25 & 2.4 \\
\hline Widowed & 7 & 0.7 \\
\hline \multicolumn{3}{|l|}{ Nationality } \\
\hline Expatriate & 736 & 69.6 \\
\hline Saudi & 322 & 30.4 \\
\hline \multicolumn{3}{|l|}{ Clinical Role } \\
\hline Consultant & 213 & 20.1 \\
\hline Assistant consultant/fellow & 52 & 4.9 \\
\hline Resident/registrar & 138 & 13.0 \\
\hline Nurse & 626 & 59.2 \\
\hline Intern/medical student & 29 & 2.7 \\
\hline \multicolumn{3}{|l|}{ Hospital Area } \\
\hline ICU & 273 & 25.8 \\
\hline ER & 110 & 10.4 \\
\hline OR & 62 & 5.9 \\
\hline Isolation ward & 63 & 6.0 \\
\hline General ward & 261 & 24.7 \\
\hline OPD & 225 & 21.3 \\
\hline Non-clinical area & 64 & 6.0 \\
\hline \multicolumn{3}{|l|}{ Hospital sector } \\
\hline Private & 174 & 16.4 \\
\hline Public/governmental & 487 & 46.0 \\
\hline University hospital & 397 & 37.5 \\
\hline \multicolumn{3}{|l|}{ Hospital specialty } \\
\hline Primary healthcare center & 123 & 11.6 \\
\hline Secondary care hospital & 196 & 18.5 \\
\hline Tertiary hospital & 739 & 69.8 \\
\hline
\end{tabular}

ICU: intensive care unit; ER: emergency room; OR: operating room; OPD: outpatient department.

Table 2

Questions regarding variant of concern B1.1.7 and number of correct answers by study participants.

\begin{tabular}{|c|c|c|}
\hline $\begin{array}{l}\text { Questions on variant of concern B.1.1.7 } \\
\text { (Correct answer) }\end{array}$ & $\begin{array}{l}\text { Incorrectly } \\
\text { answered (\%) }\end{array}$ & $\begin{array}{l}\text { Correctly } \\
\text { answered } \\
(\%)\end{array}$ \\
\hline $\begin{array}{l}\text { This new COVID-19 variant of concern } \\
\text { B.1.1.7 was first described in which } \\
\text { country? (United Kingdom) }\end{array}$ & $144(13.6)$ & $914(86.4)$ \\
\hline $\begin{array}{l}\text { Are different mutations like the COVID-19 } \\
\text { variant of concern B.1.1.7 expected to } \\
\text { happen? (True) }\end{array}$ & $277(26.2)$ & $781(73.8)$ \\
\hline $\begin{array}{l}\text { Is COVID-19 variant of concern B.1.1.7 more } \\
\text { contagious than the original virus? (True) }\end{array}$ & $249(23.5)$ & 809 (76.5) \\
\hline $\begin{array}{l}\text { Does COVID-19 variant of concern B.1.1.7 } \\
\text { cause more severe disease? (False) }\end{array}$ & $734(78.8)$ & $224(21.2)$ \\
\hline $\begin{array}{l}\text { Are the available COVID-19 vaccines in Saudi } \\
\text { effective for COVID-19 variant of concern } \\
\text { B.1.1.7? (True) }\end{array}$ & $510(48.2)$ & $548(51.8)$ \\
\hline $\begin{array}{l}\text { Are the effects of variant of concern B.1.1.7 } \\
\text { on the respiratory system worse than the } \\
\text { original COVID-19? (False) }\end{array}$ & $870(82.2)$ & $188(17.8)$ \\
\hline $\begin{array}{l}\text { Will the Variant of concern B.1.1.7 result in } \\
\text { another wave of the pandemic? (True) }\end{array}$ & $673(63.6)$ & 385 (36.4) \\
\hline $\begin{array}{l}\text { Does the appearance of variant of concern } \\
\text { B.1.1.7 mutation indicate that herd } \\
\text { immunity is occurring? (False) }\end{array}$ & $932(88.1)$ & $126(11.9)$ \\
\hline
\end{tabular}


Table 3

Descriptive bivariate analysis of HCWs' uptake of the COVID-19 vaccine ( $\mathrm{N}=1058$ )

\begin{tabular}{|c|c|c|c|c|}
\hline \multirow[t]{2}{*}{ Characteristic } & \multicolumn{2}{|c|}{ Immunization uptake $\mathbf{n}(\%)$} & \multirow[t]{2}{*}{ Test statistic } & \multirow[t]{2}{*}{ P value } \\
\hline & Not yet received & Received/ registered & & \\
\hline \multicolumn{5}{|l|}{ Gender } \\
\hline Male & $152(21.5)$ & $202(57.4)$ & \multirow[t]{2}{*}{$\chi^{2}(1)=135.6$} & \multirow[t]{2}{*}{$<0.001$} \\
\hline Female & $554(78.5)$ & $150(42.6)$ & & \\
\hline \multicolumn{5}{|l|}{ Age } \\
\hline $20-30$ years & $171(24.2)$ & $67(19.0)$ & \multirow[t]{4}{*}{$\chi^{2}(1)=12.90$} & \multirow[t]{4}{*}{0.005} \\
\hline $31-40$ years & $326(46.2)$ & $145(41.2)$ & & \\
\hline $41-50$ years & $162(22.9)$ & $101(28.7)$ & & \\
\hline$\geq 51$ years & $47(6.7)$ & $39(11.1)$ & & \\
\hline \multicolumn{5}{|l|}{ Marital state } \\
\hline Single & $177(25.1)$ & $80(22.7)$ & \multirow[t]{2}{*}{$\chi^{2}(1)=0.70$} & \multirow[t]{2}{*}{0.402} \\
\hline Married/divorced/widowed & $429(74.9)$ & $272(77.3)$ & & \\
\hline \multicolumn{5}{|l|}{ Nationality } \\
\hline Expatriate & $551(78.0)$ & $185(52.6)$ & \multirow[t]{2}{*}{$\chi^{2}(1)=72.1$} & \multirow[t]{2}{*}{$<0.001$} \\
\hline Saudi & $155(22.0)$ & $167(47.4)$ & & \\
\hline \multicolumn{5}{|l|}{ Clinical Role } \\
\hline Consultant & $93(13.2)$ & $120(34.1)$ & \multirow{5}{*}{$\chi^{2}(4)=102.4$} & \multirow[t]{5}{*}{$<0.001$} \\
\hline Assistant consultant/fellow & $31(4.4)$ & $21(6.0)$ & & \\
\hline Resident/registrar & $74(10.5)$ & $64(18.2)$ & & \\
\hline Nurse & $491(69.5)$ & $135(38.4)$ & & \\
\hline Intern/medical student & $17(2.4)$ & $12(3.4)$ & & \\
\hline \multicolumn{5}{|l|}{ Hospital ward } \\
\hline ICU & $163(23.1)$ & $110(31.2)$ & $\chi^{2}(6)=10.60$ & 0.102 \\
\hline ER & $83(11.8)$ & $27(7.7)$ & & \\
\hline OR & $4195.8)$ & $21(6.0)$ & & \\
\hline Isolation ward & $43(6.1)$ & $20(5.7)$ & & \\
\hline General ward & $179(25.4)$ & $82(23.3)$ & & \\
\hline OPD & $153(24.7)$ & $72(20.5)$ & & \\
\hline Non-clinical area & $44(6.2)$ & $20(5.7)$ & & \\
\hline Hospital sector & & & & \\
\hline Private & $122(17.3)$ & $52(14.8)$ & $\chi^{2}(2)=12.23$ & 0.002 \\
\hline Public/governmental & 345 (48.9) & $42(40.3)$ & & \\
\hline University hospital & 239 (33.9) & $158(44.9)$ & & \\
\hline Hospital specialty & & & & \\
\hline Primary healthcare center & $83(11.8)$ & $40(11.4)$ & $\chi^{2}(2)=6.10$ & 0.048 \\
\hline Secondary care hospital & $145(20.5)$ & $51(14.5)$ & & \\
\hline Tertiary hospital & $478(67.7)$ & $261(74.1)$ & & \\
\hline Had contact with patients infected with COVID & & & & \\
\hline No & $156(22.1)$ & $77(21.9)$ & $\chi^{2}(1)=0.010$ & 0.935 \\
\hline Yes & $550(77.9)$ & $275(78.1)$ & & \\
\hline Previously diagnosed with PCR-positive COVID & & & & \\
\hline No & $640(90.7)$ & $322(91.5)$ & $\chi^{2}(1)=0.194$ & 0.660 \\
\hline Yes & $66(9.3)$ & $30(8.5)$ & & \\
\hline Traveled abroad in the last 3 months & & & & \\
\hline No & $669(94.8)$ & $329(93.5)$ & $\chi^{2}(1)=0.73$ & 0.391 \\
\hline Yes & $37(5.2)$ & $23(6.5)$ & & \\
\hline Use of social media as a source of information & & & & \\
\hline No & $213(30.2)$ & $136(38.6)$ & $\chi^{2}(1)=7.62$ & $<0.001$ \\
\hline Yes & $493(69.8)$ & $216(61.4)$ & & \\
\hline GAD-7 score & $5.29(5.10)$ & $4.36(4.75)$ & $t(1056)=2.85$ & 0.004 \\
\hline Awareness of the COVID-19 VoC mean score & $3.59(1.13)$ & $4.10(1.40)$ & $\mathrm{t}(601.8)=6.03$ & $<0.001$ \\
\hline Perceived worry level about travelling abroad & $3.30(1.17)$ & $3.10(1.90)$ & $\mathrm{t}(1058)=3.10$ & 0.002 \\
\hline
\end{tabular}

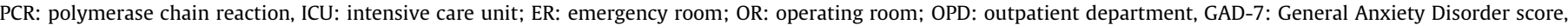
VoC: variant of concern.

Table 4

Multivariate logistic binary regression analysis of HCWs' COVID-19 immunization behavior (registering or receiving the vaccine) $(\mathrm{N}=1058$ ).

\begin{tabular}{|c|c|c|c|c|}
\hline \multirow[t]{2}{*}{ Characteristic } & \multirow[t]{2}{*}{ Multivariate aOR } & \multicolumn{2}{|l|}{$95 \% \mathrm{CI}$} & \multirow[t]{2}{*}{ P value } \\
\hline & & Lower & Upper & \\
\hline Gender $=$ Female & 0.287 & 0.206 & 0.401 & $<0.001$ \\
\hline Age above 40 years & 1.021 & 1.002 & 1.040 & 0.032 \\
\hline Marital status $=$ Married/divorced/widowed & 0.799 & 0.545 & 1.169 & 0.247 \\
\hline Nationality = Saudi & 1.918 & 1.363 & 2.698 & $<0.001$ \\
\hline Hospital ward = ICU & 1.495 & 1.083 & 2.063 & 0.014 \\
\hline Hospital sector $=$ University hospital & 1.867 & 1.380 & 2.525 & $<0.001$ \\
\hline Mean score awareness of variant of concern (range: $0-8$ points) & 1.131 & 1.002 & 1.278 & 0.047 \\
\hline GAD-7 score & 0.995 & 0.966 & 1.025 & 0.742 \\
\hline Travelled abroad in last 3 months & 1.624 & 0.889 & 2.964 & 0.114 \\
\hline Previously diagnosed with COVID-19 & 0.880 & 0.536 & 1.445 & 0.614 \\
\hline Use of social media as a source of information & 0.207 & 0.132 & 1.354 & 0.001 \\
\hline
\end{tabular}

ICU: intensive care unit, GAD-7: General Anxiety Disorder score 


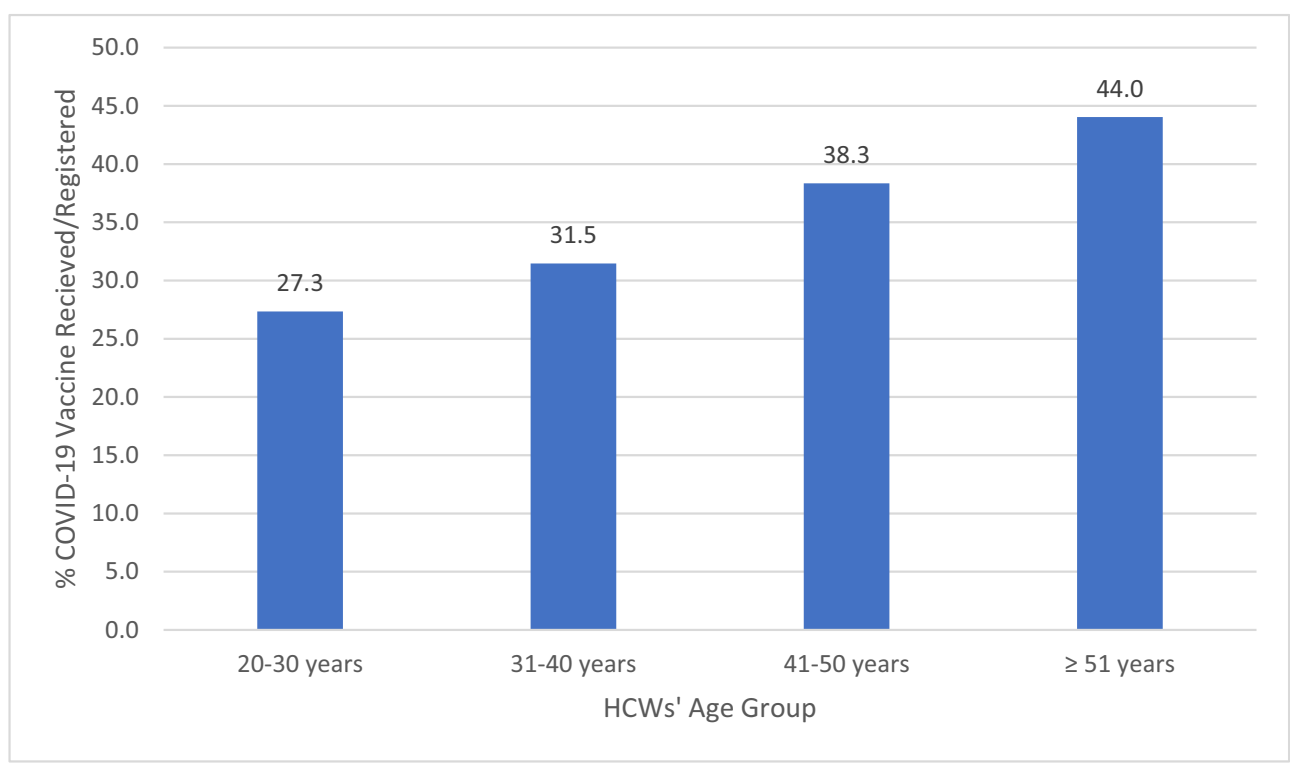

Fig. 1. The association between HCWs' age group and their model mean predicted probability of COVID-19 vaccination.

vaccine within 3 weeks of its availability. In a previous crosssectional survey to assess HCWs' COVID-19 vaccine confidence and hesitancy prior to launching a vaccine campaign in the KSA, $70 \%$ were willing to receive a vaccine once available [6]. Additionally, half of the participants indicated that they would receive the vaccine as soon as it became available, while more than one-third preferred delaying receiving it for a few months. In a study that specifically focused on vaccine acceptance according to vaccine type, only $20.9 \%$ were willing to receive BNT162b2 [6,19]. The acceptance of vaccines in KSA is variable. Previous studies showed significant hesitancy for H1N1 vaccine [20]. Another study showed that $36.6 \%$ of surveyed HCW received the seasonal influenza vaccination in 2008-2009, and only $31.1 \%$ were willing to have the H1N1 vaccine [21]. Generally, the acceptance rate of COVID-19 vaccine in the Middle East is thought to be $23-66 \%$ [22,23]. In a study from Saudi Arabia, acceptance of the COVID-19 vaccine among the general population was less than 50\% among 3101 participants [24]. An additional study showed that about $60 \%$ would take the COVID-19 vaccine [25]. Our study looked at acceptance of vaccination within the first month of vaccine rollout within HCWs, no similar studies have been done on the general public in KSA, and no data was published on vaccination uptake by $\mathrm{MoH}$ during the study period. The low vaccine uptake reported in the current study, together with HCWs' earlier reports of preferring to delay getting vaccinated, is alarming and should trigger public health officials to target these groups with campaigns to enhance their vaccine confidence and acceptance.

In the current study, two-thirds of participants were female, almost $60 \%$ were nurses, $70 \%$ were expatriates, and the majority worked at tertiary care hospitals. These findings are similar to a previous study that was conducted prior to the vaccine rollout [6]. However, that study included only $50 \%$ nurses. In this study, $69 \%$ of nurses had neither received nor registered to receive the vaccine, while half of the physicians had. This is similar to influenza vaccine uptake among HCWs, as it has been reported that physicians have significantly higher flu vaccination rates compared to nurses [26,27].

Almost all of the participants worked in clinical areas, and $80 \%$ managed COVID-19 patients. HCWs working in clinical areas other than the ICU, such as the emergency room (ER) and wards, did not converge significantly on their vaccine uptake. In an influenza vac- cine uptake study, only working in the building where the vaccination was being performed made a significant difference [28].

HCWs from university hospitals were found to be significantly more likely to receive the vaccine than those working in private and public sectors. Additionally, HCWs in tertiary healthcare settings were significantly more likely to get the vaccine than those working in primary and secondary healthcare settings. In a systematic review on influenza vaccination among HCWs, the top reason for vaccine uptake was self-protection rather than protecting patients or setting an example for their patients, with no observed difference in hospital settings [29].

A gender difference was observed in the vaccine uptake, with female HCWs being significantly less likely to receive the vaccine than male HCWs $(\mathrm{P}<0.001)$. The discrepancy between males and females in the uptake of this vaccine is interesting to address, in order to optimize vaccination for all genders. This is vital, as it is estimated that women comprise $70 \%$ of the global COVID-19 healthcare workforce [30]. Also, male sex was shown to be associated with increased death and ICU admission in a recent metaanalysis of COVID-19 patients [31]. However, anti-Spike antibodies declined faster in female than male patients in a recent French study [32]. One factor that may have influenced female HCWs to be less inclined to receive BNT162b2 is the lack of safety data from the randomized clinical trial that excluded pregnant and breastfeeding women [3], although our survey did not specifically ask women on their pregnancy/breastfeeding status or if they were planning on getting pregnant, this may certainly have been a potential reason for their lower uptake. Newly emerged preliminary data on pregnant women identified from the v-safe after vaccination health checker surveillance system, the v-safe pregnancy registry and the Vaccine Adverse Event Reporting System did not show any obvious safety signals with mRNA vaccines [33] and a retrospective cohort study of pregnant women vaccinated with BNT162b2 matched to unvaccinated women showed a significantly lower risk of SARS-CoV-2 infection [34]. These gender differences are important to further enhance our understanding of vaccine uptake and design-specific interventions.

While another study showed no effect of age on COVID-19 vaccine acceptance among the general population [35], our study revealed that HCWs over 40 years of age were more motivated to receive the vaccine. This is in contrast to a vaccine intent survey 
among nurses that showed a stronger COVID-19 vaccination intention among younger workers [7]. While older HCWs are at a higher risk of COVID-19 infection, protection of the entire healthcare workforce is crucial during this pandemic.

Saudi HCWs were found to be significantly more likely to receive the vaccine than expatriates $(\mathrm{P}<0.001)$. The KSA has made the COVID-19 vaccine available free of charge to all citizens and residents. A previous study found a disparity in the outcome of patients infected with SARS-CoV-2 in relation to gender and ethnicity [36]. In a study among blood donors in the KSA, nonSaudis were found to be more likely to have positive SARS-CoV-2 serology [37]. These differences between Saudi and expatriates deserve further study in order to understand the factors contributing to this disparity, which could allow for strategies and communication plans to alleviate the risk of exposure to SARS-CoV-2 and enhance the acceptance of the COVID-19 vaccine among the population.

HCWs' clinical role was correlated with their uptake of the vaccine in the bivariate analysis. Nurses were found to be significantly less inclined to receive the vaccine than physicians and other professionals $(\mathrm{P}<0.001)$, which is similar to a previous study from the KSA [6]. The multivariate analysis did not show any significant differences between location with the exception of ICU staff, who were significantly more inclined to receive the vaccine $(\mathrm{aOR}=1.495, \mathrm{P}=0.014)$. HCWs are more likely to acquire vaccine-preventable diseases, with $20 \%$ of HCWs contracting influenza annually, recent reports showed low influenza vaccine uptake among doctors and nurses (56.5\% and 34.8\% acceptance rate, respectively) [25].

Interestingly, no significant differences in vaccine uptake were found between HCWs who managed COVID-19 patients compared to those who did not or between HCWs with previous COVID-19 infections compared to those without. The low COVID-19 vaccine uptake rate in the middle of a pandemic is alarming, and efforts should focus on increasing vaccine acceptance and uptake to match the speed of the pandemic.

The VoC-202012/01 emerged in December 2020, resulting in new travel restrictions [38]. However, there is evidence that the BNT162b2 vaccine is effective against this variant based on invitro studies [39]. In this study, vaccine uptake was not influenced by HCWs' travel history. However, the overall sample size of returning travelers was small and may not be representative. Information on evolving variants are emerging in various countries [40], and concerns regarding the vaccine's efficacy against these variants may hinder vaccine uptake. This is a concerning situation that warrants further study.

Even before the COVID-19 pandemic, vaccination was considered an emotionally charged topic in many cultures [41]. However, vaccine hesitancy is common and includes people who have not yet rejected vaccination but do not trust the institutions connected to the vaccine [42]. Current recommendations suggest not only to make a safe and effective vaccine available but also deep engagement of around the human element to build public trust in any vaccine [43]. This highlights the importance of addressing societal concerns and fears to ensure a vaccination campaign's success [44]. Personal worries and baseline anxiety should not be neglected as these could trigger vaccine refusal in the community via the butterfly effect. Findings in the current study highlight how HCWs, especially those with lower GAD-7 scores, were more likely to accept the new vaccine. It also provides a glimpse of the relationship between higher awareness (in this case, of the new variant) and the likelihood of considering vaccination.

The use of social media for information could greatly affect HCWs' and the general populations' COVID-19 vaccine acceptance. While some studies did not find associations between willingness to vaccinate and social media use $[35,45]$, others found a higher vaccination willingness among respondents from the general population who did not rely on social media for COVID-19 information $[23,46]$. In one study the most commonly used platforms to get information about COVID-19 in Saudi Arabia were the internet and social media (85.8\%) [47]. And another study showed that the most common source of information for COVID-19 in Saudi Arabia were official government social media, and Twitter [48]. Other sources of getting information regarding COVID-19 in Saudi Arabia includes text messages, television, and the official Saudi MOH call center (the 937 number) [49]. Other studies also showed dependence on the Saudi and US Centers for Disease control and preventions [50], or the official websites of the Saudi MOH and the World Health Organization (WHO) [51]. One study assessed the attitudes towards COVID-19 vaccines using the Vaccine Conspiracy Belief Scale and showed higher conspiracy beliefs among respondents who relied on social media platforms as their main source of information [23].

\subsection{Study limitations and strengths/future potential}

This study is subject to the limitations of cross-sectional surveys, including sampling, response, recall biases, the use of a convenience sample that may have resulted in the imbalance between gender representation in the current study. Additional studies are needed to confirm the findings of this study and to include more balanced sample in relation to the gender. Another limitation is that the vaccine at the time of the survey was new and many HCWs may not have been educated about it, as well as selfreport which is a limitation for survey questions. While this work did not explore the reasons why HCWs did not register for the vaccine and did not ask women about pregnancy, it presents their initial vaccination acceptance, which needs to be explored in future studies. It should be noted that HCWs' perceptions and vaccine hesitancy may differ from one country to another.

\subsection{Conclusion}

This study observed a low level of COVID-19 vaccine enrollment among HCWs during the first month of vaccine rollout in one of the first countries to roll it out. Public health officials should scale up their efforts to increase vaccine acceptance and uptake among HCWs to match the speed of the growing pandemic. Optimizing protection of HCWs through vaccination and encouraging them to subsequently recommend vaccination to their patients is vital to curbing this global crisis.

\section{Declaration of Competing Interest}

The authors declare that they have no known competing financial interests or personal relationships that could have appeared to influence the work reported in this paper.

\section{References}

[1] Lurie N, Saville M, Hatchett R, Halton J. Developing covid-19 vaccines at pandemic speed. N Engl J Med 2020;382(21):1969-73.

[2] National Academies of Sciences E, and Medicine. Framework for equitable allocation of COVID-19 vaccine; 2020.

[3] Polack FP, Thomas SJ, Kitchin N, Absalon J, Gurtman A, Lockhart S, et al. Safety and efficacy of the BNT162b2 mRNA covid-19 vaccine. N Engl J Med 2020;383 (27):2603-15.

[4] Baden LR, El Sahly HM, Essink B, Kotloff K, Frey S, Novak R, et al. Efficacy and safety of the mRNA-1273 SARS-CoV-2 vaccine. N Engl J Med 2021;384 (5):403-16.

[5] Voysey M, Clemens SAC, Madhi SA, Weckx LY, Folegatti PM, Aley PK, et al. Safety and efficacy of the ChAdOx1 nCoV-19 vaccine (AZD1222) against SARSCoV-2: an interim analysis of four randomised controlled trials in Brazil, South Africa, and the UK. The Lancet 2021;397(10269):99-111. 
[6] Barry M, Temsah M-H, Alhuzaimi A, Alamro N, Al-Eyadhy A, Aljamaan F, et al. COVID-19 vaccine confidence and hesitancy among healthcare workers: a cross-sectional survey from a MERS-CoV experienced nation. medRxiv 2020.

[7] Kwok KO, Li K-K, Wei WI, Tang A, Wong SYS, Lee SS. Influenza vaccine uptake, COVID-19 vaccination intention and vaccine hesitancy among nurses: a survey. Int J Nurs Stud 2020;114:103854.

[8] Reiter PL, Pennell ML, Katz ML. Acceptability of a COVID-19 vaccine among adults in the United States: how many people would get vaccinated? Vaccine 2020;38(42):6500-7.

[9] Head KJ, Kasting ML, Sturm LA, Hartsock JA, Zimet GD. <? covid19?> A national survey assessing SARS-CoV-2 vaccination intentions: implications for future public health communication efforts. Sci Commun 2020;42(5):698-723.

[10] WHO. COVID-19 Saudi Arabia Situation.

[11] SFDA. Approved registration of Pfizer-BioNTech COVID-19 Vaccine in the Kingdom of Saudi Arabia; 2020.

[12] Craven J. COVID-19 vaccine tracker. Regulatory Affairs Professionals Society; 2020.

[13] Bloomberg. COVID-19 Vaccine Tracker.

[14] Al-Hanawi MK, Khan SA, Al-Borie HM. Healthcare human resource development in Saudi Arabia: emerging challenges and opportunities-a critical review. Public Health Rev 2019;40:1.

[15] Temsah MH, Alhuzaimi AN, Alamro N, Alrabiaah A, Al-Sohime F, Alhasan K, et al. Knowledge, attitudes, and practices of healthcare workers during the early COVID-19 pandemic in a main, academic tertiary care centre in Saudi Arabia. Epidemiol Infect 2020:1-29.

[16] Temsah M-H, Al-Sohime F, Alamro N, Al-Eyadhy A, Al-Hasan K, Jamal A, et al. The psychological impact of COVID-19 pandemic on health care workers in a MERS-CoV endemic country. J Infect Public Health 2020;13(6):877-82.

[17] Temsah MH, Barry M, Aljamaan F, Alhuzaimi AN, Al-Eyadhy A, Saddik B, et al. SARS-CoV-2 B.1.1.7 UK variant of concern lineage-related perceptions, COVID19 vaccine acceptance and travel worry among healthcare workers. Front Public Health 2021;9:686958.

[18] Xiaoming Xu, Ming Ai, Su H, Wo W, Jianmei C, Oi Z, et al. The psychological status of 8817 hospital workers during COVID-19 Epidemic: a cross-sectional study in Chongqing. J Affect Disord 2020;276:555-61.

[19] Temsah MH, Barry M, Aljamaan F, Alhuzaimi A, Al-Eyadhy A, Saddik B, et al. Adenovirus and RNA-based COVID-19 vaccines' perceptions and acceptance among healthcare workers in Saudi Arabia: a national survey. BMJ Open 2021;11:e048586.

[20] Barry MA, Aljammaz KI, Alrashed AA, Weina PJ. Knowledge, attitude, and barriers influencing seasonal influenza vaccination uptake. Can J Infect Dis Med Microbiol 2020;2020:1-6.

[21] Al-Tawfiq JA. Willingness of health care workers of various nationalities to accept H1N1 (2009) pandemic influenza A vaccination. Ann Saudi Med 2012;32(1):64-7.

[22] Salali GD, Uysal MS. COVID-19 vaccine hesitancy is associated with beliefs on the origin of the novel coronavirus in the UK and Turkey. Psychol Med 2020;13.

[23] Sallam M, Dababseh D, Eid H, Al-Mahzoum K, Al-Haidar A, Taim D, et al. High rates of COVID-19 vaccine hesitancy and its association with conspiracy beliefs: a study in Jordan and Kuwait among other Arab countries. Vaccines (Basel) 2021;9.

[24] Magadmi RM, Kamel FO. Beliefs and barriers associated with COVID-19 vaccination among the general population in Saudi Arabia. BMC Public Health $2021 ; 21: 1438$.

[25] Elharake JA, Galal B, Alqahtani SA, Kattan RF, Barry MA, Temsah M-H, et al. COVID-19 vaccine acceptance among health care workers in the Kingdom of Saudi Arabia. Int J Infect Dis 2021;109:286-93.

[26] Martinello RA, Jones L, Topal JE. Correlation between healthcare workers' knowledge of influenza vaccine and vaccine receipt. Infect Control Hosp Epidemiol 2003;24(11):845-7.

[27] Schult TM, Awosika ER, Hodgson MJ, Hirsch PR, Nichol KL, Dyrenforth SR, et al. Innovative approaches for understanding seasonal influenza vaccine declination in healthcare personnel support development of new campaign strategies. Infect Control Hosp Epidemiol 2012;33(9):924-31.
[28] Bautista D, Vila B, Usó R, Téllez M, Zanón V. Predisposing, reinforcing, and enabling factors influencing influenza vaccination acceptance among healthcare workers. Infect Control Hosp Epidemiol 2006;27(1):73-7.

[29] Hollmeyer HG, Hayden F, Poland G, Buchholz U. Influenza vaccination of health care workers in hospitals-a review of studies on attitudes and predictors. Vaccine 2009;27(30):3935-44.

[30] Miyamoto I. COVID-19 Healthcare workers: 70\% are women: Daniel K. Inouye Asia-Pacific Center for Security Studies; 2020.

[31] Peckham H, de Gruijter NM, Raine C, Radziszewska A, Ciurtin C, Wedderburn LR, et al. Male sex identified by global COVID-19 meta-analysis as a risk factor for death and ITU admission. Nat Commun 2020;11:1-10.

[32] Grzelak L, Velay A, Madec Y, Gallais F, Staropoli I, Schmidt-Mutter C, et al. Sex differences in the evolution of neutralizing antibodies to SARS-CoV-2. J Infect Dis 2021.

[33] Shimabukuro TT, Kim SY, Myers TR, Moro PL, Oduyebo T, Panagiotakopoulos L, et al. Preliminary findings of mRNA covid-19 vaccine safety in pregnant persons. N Engl J Med 2021;384(24):2273-82.

[34] Goldshtein I, Nevo D, Steinberg DM, Rotem RS, Gorfine M, Chodick G, et al. Association between BNT162b2 vaccination and incidence of SARS-CoV-2 infection in pregnant women. Jama 2021.

[35] Alley SJ, Stanton R, Browne M, To QG, Khalesi S, Williams SL, et al. As the pandemic progresses, how does willingness to vaccinate against COVID-19 evolve? Int J Environ Res Public Health 2021;18.

[36] Tirupathi R, Muradova V, Shekhar R, Salim SA, Al-Tawfiq JA, Palabindala V. COVID-19 disparity among racial and ethnic minorities in the US: a cross sectional analysis. Travel Med Infect Dis 2020;38:101904.

[37] Banjar A, Al-Tawfiq JA, Alruwaily A, Alserehi H, Al-Qunaibet A, Alaswad R, et al. Seroprevalence of antibodies to SARS-CoV-2 among blood donors in the early month of the pandemic in Saudi Arabia. Int J Infect Dis 2021.

[38] ECDC. Risk related to spread of new SARSCoV-2 variants of concern in the EU/ EEA.

[39] Xie X, Zou J, Fontes-Garfias CR, Xia H, Swanson KA, Cutler M, et al. Neutralization of N501Y mutant SARS-CoV-2 by BNT162b2 vaccine-elicited sera. bioRxiv 2021.01.;07(425740).

[40] CDC. New COVID-19 Variants.

[41] Ozawa S, Stack ML. Public trust and vaccine acceptance-international perspectives. Human Vaccines Immunother 2013;9(8):1774-8.

[42] Yaqub O, Castle-Clarke S, Sevdalis N, Chataway J. Attitudes to vaccination: a critical review. Soc Sci Med 2014;112:1-11.

[43] Schoch-Spana M, Brunson EK, Long R, Ruth A, Ravi SJ, Trotochaud M, et al. The public's role in COVID-19 vaccination: Human-centered recommendations to enhance pandemic vaccine awareness, access, and acceptance in the United States. Vaccine 2020

[44] Ropeik D. How society should respond to the risk of vaccine rejection. Hum Vaccin Immunother 2013;9(8):1815-8.

[45] Temsah M-H, Al Huzaimi A, Alrabiaah A, Alamro N, Al-Sohime F, Al-Eyadhy A, et al. Changes in healthcare workers' knowledge, attitudes, practices, and stress during the COVID-19 pandemic. Medicine 2021;100:e25825.

[46] Ruiz JB, Bell RA. Predictors of intention to vaccinate against COVID-19: results of a nationwide survey. Vaccine 2021;39(7):1080-6.

[47] Alnasser AHA, Al-Tawfiq JA, Al-Kalif MSH, Shahadah RFB, Almuqati KSA, AlSulaiman BSA, et al. Public knowledge, attitudes, and practice towards COVID19 pandemic in Saudi Arabia: a web-based cross-sectional survey. Med Sci (Basel) 2021;9.

[48] Alnasser AHA, Al-Tawfiq JA, Al Kalif MSH, Alobaysi AMA, Al Mubarak MHM, Alturki HNH, et al. The positive impact of social media on the level of COVID19 awareness in Saudi Arabia: a web-based cross-sectional survey. Infez Med 2020;28:545-50.

[49] Almuzaini Y, Mushi A, Aburas A, Yassin Y, Alamri F, Alahmari A, et al. Risk communication effectiveness during COVID-19 pandemic among general population in Saudi Arabia. Risk Manag Healthc Policy 2021;14:779-90.

[50] Hassounah M, Raheel H, Alhefzi M. Digital response during the COVID-19 pandemic in Saudi Arabia. J Med Internet Res 2020;22:e19338.

[51] Akhtar S, Alharbi AH, Jamal QMS, Nair KS. Knowledge and source of information of COVID-19 among students of health informatics, Qassim University, Saudi Arabia. J Pharm Res Int 2020:28-35. 